\title{
MM-PBSA and the importance of the dielectric constant for kinase drug design
}

\author{
Melanie Schneider ${ }^{1}$ and Gilles Labesse ${ }^{1}$ \\ ${ }^{1}$ Centre de Biochimie Structurale (CBS), CNRS, INSERM, Univ Montpellier, 34090 Montpellier, France.
}

\begin{abstract}
Predicting the interactions between a set of small molecules and its target plays a critical role in drug discovery and development. Especially in later stages of the drug design process, when a reduced set of molecules is in focus, reliable and accurate binding affinity estimations are important for targeted modifications of given lead molecules. Current limitations in affinity prediction originate from the lack of accurate estimates for solvation energy and entropy. MM-PBSA and the related MM-GBSA aim at providing better estimates. From our studies we infer that the common approach using one dielectric constant for the binding pocket may be misleading (here in the case of a kinase), especially when designed ligands/drugs contain charges. Thus, a range of selected values for the solute dielectric constant is preferred for better and more reliable comparisons.
\end{abstract}

Keywords: MM-PBSA, drug design, kinase, B-RAF, dielectric constant

\section{INTRODUCTION}

While a relative and approximate ranking of the stability of different complexes might be sufficient for an initial screening protocol, a finer and more accurate evaluation of the binding free energy may be necessary for a fine tuning in later stages of drug design. Free energy estimates require simulation of complex flexibility and desolvation upon binding of both partners in order to deduce the entropy term instead of a simple extrapolation of the enthalpy term with a very rough and partial prediction of the entropy part as in usual and quick affinity prediction methods. For the most accurate methods, very long simulations are required and limit their use. If accurate energies are needed, the methods of choice are sophisticated MD-based calculations, such as thermodynamic integration (TI) $[1,2]$ and free energy perturbation (FEP) [3]. Since they are computationally very expensive, extremely time-consuming and exhaustive conformational and statistical sampling is needed to obtain converged results, they are not widely used in structure-based drug design. Among the approximate methods, there are the linear interaction energy (LIE) [4], the molecular mechanics Poisson-Boltzmann surface area (MM-PBSA) [5] and the related molecular mechanics generalized Born surface area (MM-GBSA) methods [6]. LIE is a semi-empirical method, based on the assumption that the binding free energy between the ligand and the receptor can be modelled as a linear-response combining weighted electrostatic and Van der Waals interactions with coefficients varying for different systems $[4,7]$. Unlike the LIE method, MM-PBSA and MM-GBSA do not employ empirical parameters within their calculations, which makes them promising methods for ranking very different compounds. They both use molecular mechanics force fields with continuum solvent models. The GB equation is simply an approximation of the PB equation [8], resulting in an increased calculation speed (about 5 times faster), but often goes along with an accuracy tradeoff $[9,10,11]$. They are both frequently used in structure-based drug design due to their rather high accuracy and relative high computational efficiency. Another advantage is that they have no varying parameters for different protein-ligand systems while using sets of physically well-defined energy terms and they do not require training set calculations.

It has been previously reported that using MM-PBSA long MD simulations seem not to result in better predictions and short MD simulations can be adequate in calculating binding affinities $[12,13]$. In order to achieve a higher precision it has been suggested to run many short independent simulations (produced by e.g. replicate sampling) instead of a single long one, which should avoid underestimation of the uncertainty [14]. Additionally, if one is only interested in the relative order of binding affinities, the ranking of compounds with similar structures and binding modes, the entropy contribution to the binding free energy can be omitted, which is often recommended as it reduces the computational cost and avoids adding an additional non-negligible error margin. It has been found that MM-PB/GBSA performances generally vary with the tested system and also depend on the used force fieldand the solute dielectric constant $[12,15,8]$.

Here, we wanted to reassess the use of MM-PBSA for fine ranking of a drug, dabrafenib, and its known metabolites in aim at predicting a potential impact of its pharmacokinetics, its metabolization on its efficacy on its primary target the protein-kinase BRAF.

Dabrafenib [16] is a BRAF kinase inhibitor, which inhibits BRAF V600 mutation-positive cancer cell growth. It is an FDA approved drug indicated for the treatment of adult patients with unresectable or metastatic melanoma with a BRAF V600 mutation $[17,18]$ and as combination therapy since recently also for metastatic non-small cell lung cancer harboring BRAF V600E mutations [19]. Despite improved response rates and overall survival of BRAF-V600 mutant cancer patients, resistance is rapidly acquired, resulting in a relapse of most patients within a year [20]. This effect may be partially due to the fast metabolism of dabrafenib (with a half-life of $\sim 5$ hours [21]). There are three major metabolites of dabrafenib that have been identified with potential pharmacological effects: hydroxy-dabrafenib (HDB), carboxy-dabrafenib (CDB), and desmethyl-dabrafenib (DDB), whereas HDB appears to contribute significantly to the pharmacological activity [22]. 


\section{METHODS}

\subsection{Structure preparation and modelling}

The crystal structure of BRAF with dabrafenib as co-crystallized ligand, PDB-ID: 4XV2, was downloaded from the RCSB protein data bank (PDB). The protomeric structure (chain A) was prepared for MD with an in-house Python script using Modeller [23] for modelling missing residues to match the canonical sequence (UniProt identifier: P15056-1), but for the position V600 mutated to E. Here, the coordinates for atoms present in structure $4 \mathrm{XV} 2$ are kept fixed and only the missing loops (plus one adjacent residue, to avoid unrealistic geometries caused by ambiguous termini atom positions) are modelled. The complexes with the three metabolites CDB, HDB, and DDB are generated by docking them with PLANTS [24] into the previously generated complete protein structure with dabrafenib as an anchor. Hydrogen atoms of the respective ligands were modeled with OpenBabel at $\mathrm{pH}$ 7. This results in zero net charge for $\mathrm{DB}, \mathrm{HDB}$, and DDB and a negative net charge for $\mathrm{CDB}$, due to the deprotonated carboxy group.

\subsection{Electrostatic potential and dielectric constant maps}

The BRAF-DB complex structure was used to calculate electrostatic potential maps and dielectric maps with DelPhi $[25,26]$. DelPhi is a free command line tool that calculates the electrostatic potential for biomolecules by solving the Poisson Boltzmann equation.

\subsection{Molecular dynamics simulation}

All simulations were carried out with Gromacs 2018 [27]. The ligand topologies were generated using the ACPYPE/ANTECHAMBER [28] program of AmberTools17 [29] with partial charges generated by the empirical charge model AM1-BCC. The ligands parameters are based on the General Amber Force Field (GAFF) and the Amber FF14SB force field was employed for the proteins. Each complex was solvated in a TIP3P water dodecahedral box, with periodic boundary conditions and a minimum distance of $1.0 \mathrm{~nm}$ from the surface of the complex to the edge of the box. Each system was neutralized by adding $\mathrm{NA}+$ and $\mathrm{Cl}$ - ions to physiological concentration of $153.6 \mathrm{mM}$. A completely free steepest descent energy minimization for 2000 steps was followed by a 100-ps NVT equilibration and a 100-ps NpT equilibration with Parrinello-Rahman pressure coupling. NVT and NpT equilibrations were performed at a reference temperature of $300 \mathrm{~K}$ with ligand restraints of $1000 \mathrm{~kJ} /-$ $\mathrm{mol} \mathrm{nm}{ }^{2}$ in $\mathrm{x}, \mathrm{y}, \mathrm{z}$ directions. Finally, $50 \mathrm{~ns}$ unrestrained production runs were performed with a $2 \mathrm{fs}$ time-step in the NpT ensemble and snapshots were saved every $10 \mathrm{ps}$. For each of the four ligands (DB, $\mathrm{CDB}, \mathrm{HDB}$, and DDB) five replica simulations were run with different randomly assigned initial velocities, resulting in a total of 250 ns simulation per ligand.

\subsection{MM-PBSA calculation}

For MM-PBSA calculation the 50-ns MD production trajectories were reduced to 501 frames each, by extracting a frame every 100 ps. The resulting snapshots of the MD simulations were utilized for post-processing free energies by the single-trajectory MM-PBSA method implemented in g_mmpbsa. Six different dielectric constants $(\epsilon=(2,4,6,8,12,20))$ were used for the binding pocket, while the solution dielectric constant was kept constant at $\epsilon_{s}=80$. Calculations are performed based on a homogeneous medium with a range of dielectric constants for the solute, an ionic strength of 153.6 $\mathrm{mM}$, an ionic radius of $0.95 \AA$ for positive charged ions and 1.81 $\AA$ for negative charged ions, and a solvent probe radius of $1.4 \AA$. An example configuration file for g_mmpbsa is provided within the supplements (Listing 1). Other parameters influencing the grid dimensions of the calculation, such as 'cfac', 'gridspace' and 'fadd' were varied from suggested defaults (1.5, 0.5 and 10 , respectively) showing only marginal variations in the results and therefore not further changed.

Analysis and visualization is performed with provided scripts from the g_mmpbsa package [30], Chimera [31], PyMol [32] and Python scripts.

\section{RESULTS}

With this study we provide a basis for important considerations when employing MM-PBSA based affinity estimations on kinases. The oncogenic protein kinase BRAF-V600E together with the clinical drug dabrafenib serve as example for pointing out methodical issues that can arise when computing affinities in standard drug design projects.

As the binding mode of dabrafenib in BRAF-V600E is experimentally known (PDB-ID: 4XV2), we take advantage of this complex for further calculations and use it as template for docking the dabrafenib metabolites. Unfortunately the experimental structure is not complete and for chain A the missing loop residues (432-448, $488,489,597-614,627-631,721-723$ ) had to be modelled.

\subsection{Electrostatic potential and dielectric constant distribution of BRAF kinase}

Electrostatics plays an important role in regulating interactions between biological macromolecules. The electrostatic potential map of the protein-ligand complex BRAF-dabrafenib at $\mathrm{pH} 7$ shows remarkable variations at different slicing depths within the binding pocket, whereas the values within protein stay rather constant (see Figure 1).

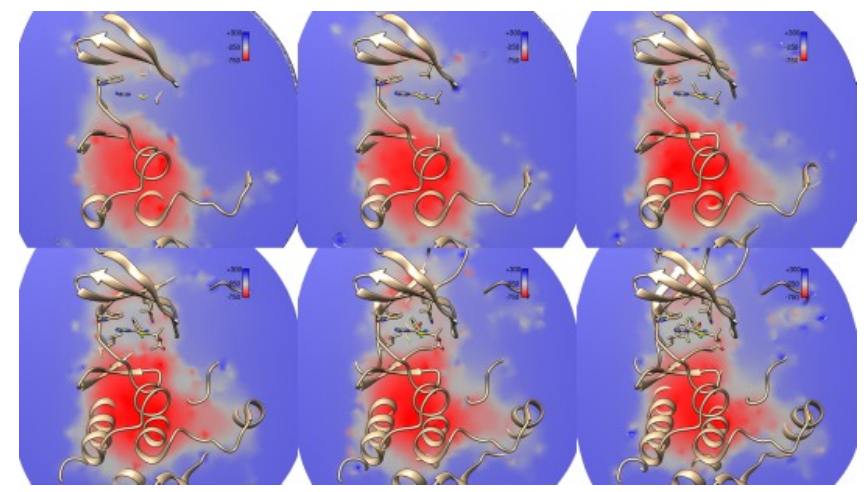

Figure 1. Electrostatic potential $(\phi)$ map calculated on the protein-ligand complex structure. Six consecutive slices through the protein that sample the depth of the binding pocket, where the coloring shows the electrostatic potential at the slicing surface. The $\phi$-map is calculated with DelPhi and visualized with Chimera. 
The dielectric constant map equally points out variations inside the binding pocket in contrast to the protein interior (compare Figure 2). In particular, the tri-methyl moiety of dabrafenib is pointing outside the binding pocket and lies outside the cutoff where an epsilon value of 80 is reached (see Figure 2 closeup on binding pocket). This indicates that it is completely solvent accessible and should be considered as solvated.

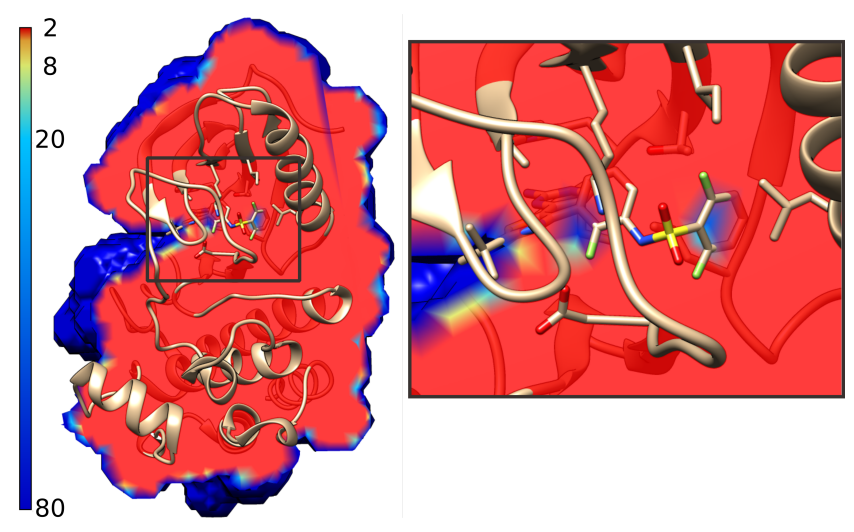

Figure 2. Dielectric constant $(\epsilon)$ map calculated on the protein-ligand complex structure with a complete view of the complex (left) and a close-up on the ligand (right). The $\epsilon$-map is calculated with DelPhi and visualized with Chimera.

\subsection{MM-PBSA, the dielectric constant and ligand charges}

MM-PBSA binding affinity calculations based on 50ns MD simulations for dabrafenib (DB) and its metabolites carboxy-dabrafenib (CDB), desmethyl-dabrafenib (DDB), and hydroxy-dabrafenib (HDB) with BRAF-V600E (see Figure 3) and with BRAF-WT with a structured, helical activation loop (see Figure S2) are dependant on the solute dielectric constant $(\epsilon)$. Especially the charged CDB shows an inverted behaviour compared to the other three molecules. Whereas the binding energy for DB, DDB and HDB gradually decreases with increasing $\epsilon$ (ranging from 2 to 20), $\mathrm{CDB}$ has an extremely low energy at $\epsilon=2$, which rapidly increases when shifting to a slightly higher $\epsilon=4$, but stays constant from $\epsilon=8$ onward.

3.2.1 Energetic contribution of protein residues to ligand binding To investigate the reason for the extreme discrepancies between calculations at different dielectric constants residue-wise energetic contributions to ligand binding are investigated using the free energy decomposition scheme of g_mmpbsa. For visualization the energies given as kJ per mole are mapped onto the structure (see Figure 4 and S1). The energetic contributions per residue along the protein sequence between a dielectric constant of 2 and 8 (shown in Figure 5) highlights extreme discrepancies for $\mathrm{CDB}$, whereas only the $\mathrm{CDB}$ pattern with $\epsilon=8$ agrees with the patterns for DB, DDB and HDB, which are very similar. Therefore, calculations performed with a dielectric constant of 2 are considered as untrustworthy and protein residues with most important energetic contributions are compared between the DB and its metabolites at $\epsilon=8$ (see Figure 6). The contributions appear to be highly similar, except for Lys483, which shows

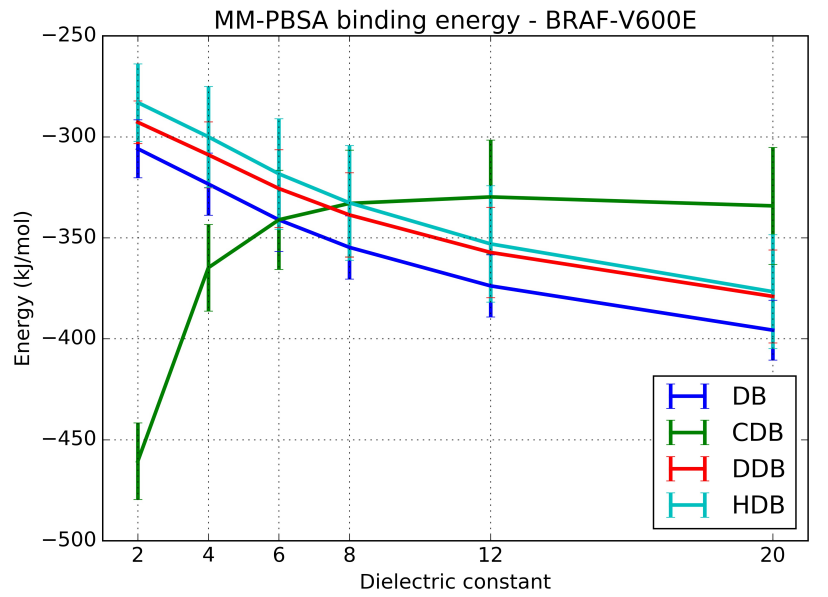

Figure 3. Averaged MM-PBSA binding energies (only enthalpic contribution) for BRAF-V600E with dabrafenib (DB) and its metabolites carboxydabrafenib (CDB), desmethyl-dabrafenib (DDB), and hydroxy-dabrafenib (HDB). The averages are calculated for each ligand based on 2505 complex conformations from five replica MD trajectories at six different dielectric constants $(2,4,6,8,12$, and 20$)$. The error bars are standard deviations across the five replica MD trajectories for each dielectric constant.

increased variations and tends to more favorable energies for DB than its metabolites.
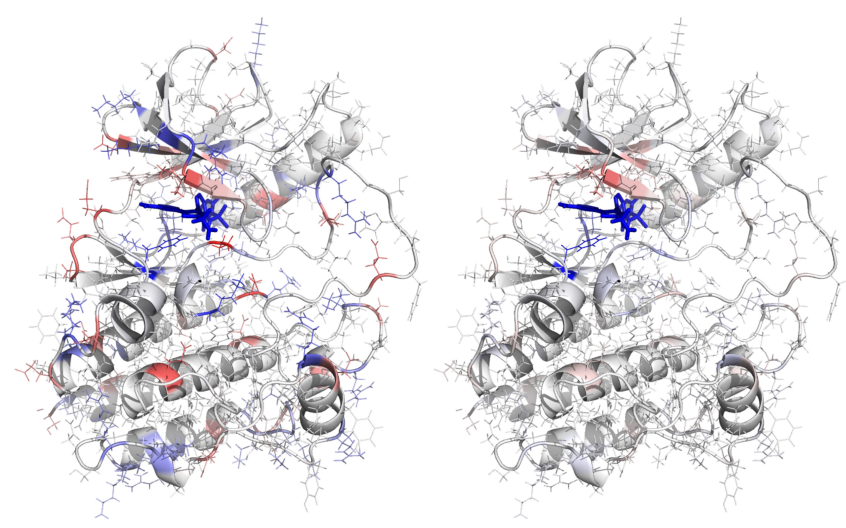

Figure 4. Energetic contribution of protein residues to ligand binding for the charged metabolite carboxy-dabrafenib (CDB) calculated with different dielectric constants of 2 (left) and 8 (right). Color coding = blue-white-red, with a minimum of -33 and a maximum of $+33 \mathrm{~kJ} / \mathrm{mol}$ (visualized with PyMol)

\subsubsection{Complex evaluation by scoring function DSX}

As external validation of the binding poses of the docked metabolites CDB, DDB and HDB the knowledge-based scoring function DSX (via DSX-online [33]) was used to evaluate the docked complexes that served as starting structures for MD simulations. Providing a score for protein-ligand complexes together with a visualization of the per-atom score contributions DSX-online allows 

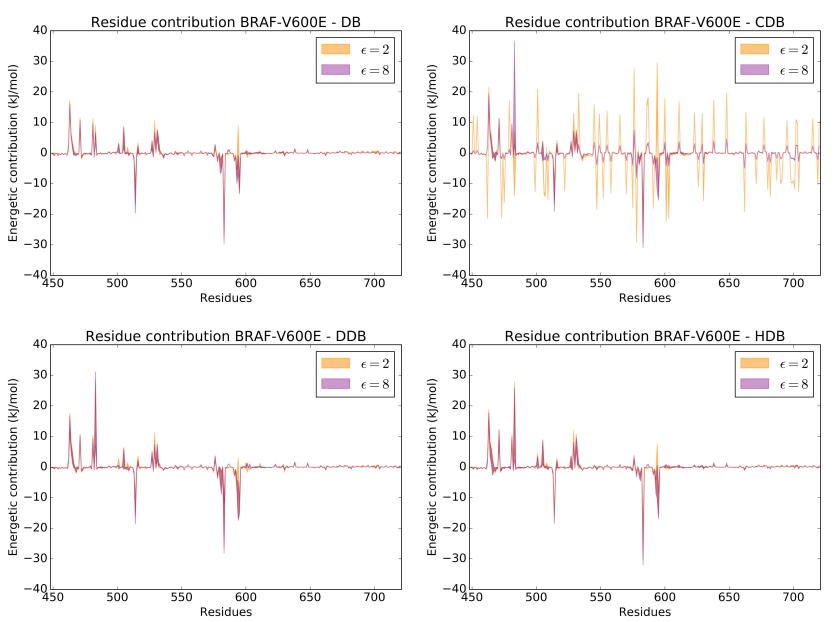

Figure 5. Energetic contribution of protein residues to ligand binding for $\mathrm{DB}$ and its metabolites $\mathrm{CDB}, \mathrm{DDB}$ and $\mathrm{HDB}$, calculated with dielectric constants of 2 and 8 .

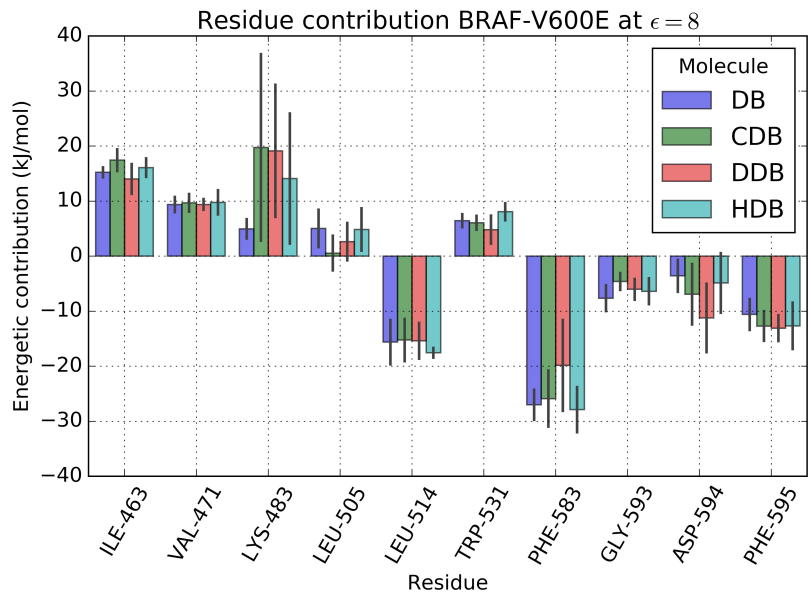

Figure 6. Protein residues with most important energetic contributions (absolute value larger than $10 \mathrm{~kJ} / \mathrm{mol}$ for at least one simulation) to ligand binding for $\mathrm{DB}$ and its metabolites $\mathrm{CDB}, \mathrm{DDB}$ and $\mathrm{HDB}$, calculated with a dielectric constant of 8 . The bar heights are the average values across the 5 replicas and the error

for investigating possible reasons for binding discrepancies. DSX scoring (using CSD potentials) provides the following ranking (from best to worst, with respective scores):

$$
\text { DB (-168.6) - HDB (-137.7) - CDB (-135.7) - DDB (-124.9). }
$$

The four molecules are scored highly favorable, very similar and the visualization of the per-atom score contributions showed only marginal variations for the moieties differing between the molecules (see Figure S3). Only minor unfavorable distances were detected between a few atoms of the identical parts of the metabolites and surrounding protein residues (that are supposedly due to tiny pose variations upon docking).

The evaluation by DSX suggests that the metabolites have highly similar binding affinities, which is equally the case for the MMPBSA calculations with a dielectric constant of 8 (see Figure 3 and S2) and therefore, additionally confirms the parameter choice $\epsilon=8$ for the protein kinase.

\subsubsection{Affinity prediction by docking and machine learning}

In order to further evaluate the proper ranking of the four compounds (DB, CDB, DDB and HDB), we applied a second completely independent affinity prediction method based on machine learning. The method is described in details in a previous publication [34]. Training of the random forest machine learning algorithm in regression mode was performed based on the ligand dataset BRAF-V600E (with annotated IC50 affinity measures - 2193 molecules) from BindingDB (2018). The method relies on data from multi-structure docking and pose evaluation of the ligand dataset on the @ TOME server [35] taking into account all available BRAF structures, and ligand-based molecular descriptors. The machine learning method predicted the following affinity ranking (from highest to lowest) with pIC50 values ([- $\left.\left.\log _{10}(M)\right]\right)$ :

$$
\text { DB (8.42) - HDB (8.19) - CDB (8.05) - DDB (8.00). }
$$

Remarkably, the machine learning based ranking is the same as for the DSX evaluation, also predicting highly similar binding affinities for the metabolites and an increased affinity for DB. This again, confirms the validity of the choice of the dielectric constant $(\epsilon=8)$, at with this tendency is equivalently reproduced.

\subsubsection{Comparison with reported affinity measures from literature}

GlaxoSmithKline published studies on the activity of their drug Dabrafenib and the three mayor metabolites [36, 37]. Interestingly, measured half-lives for $\mathrm{CDB}$ and $\mathrm{DDB}$ were longer than for DB and HDB [36]. The study of Ellens et al. [37] reports that, based on in vitro antiproliferative IC50 measures, HDB and DDB should be potent inhibitors of BRAF-V600E, slightly less active than DB whereas the activity of $\mathrm{CDB}$ is largely reduced. Comparison of the affinity measures with the affinity ranking using MM-PBSA suggests that high values of the dielectric constant $(\epsilon>8)$ are appropriate to obtain the equivalent ranking of DB - HDB/DDB $\mathrm{CDB}$, from best to worst (compare Figure 3).

\section{DISCUSSION}

Most biological processes are influenced or even governed by electrostatic effects. Structure-function correlations in general and ligand-receptor interactions in particular are heavily dependant on accurate electrostatic calculations. Just as the electrostatic contribution to the solvation / desolvation process has proved to be of paramount importance. However, the need for discriminatory case studies has not received the attention it deserves. The PB and GB models, provide very performant tools for modelling the effect of the solvent around the protein and they are widely used techniques for binding affinity estimation. The electrostatic contribution is modeled here as a dielectric linear response to the electric field generated by the atomic charges. There have been several previously reported promising results with excellent correlations with experimental data $[38,39,40]$. 
It has been shown that in implicit solvent simulations that use PB forces employing a dielectric constant of 1.0 (as used in many force fields) the resulting MD trajectories do not always preserve native structures (using different protocols and programs). To reduce solvation forces, a common technique is to raise the dielectric constant [6]. The dielectric constant for a protein has traditionally been estimated below, or at around 4 [41, 42], but can also be significantly larger, as large as 10, in sites of catalytic importance [43]. The optimal value differs between systems and are for example set to 4 [44] or even to 17 [45]. For implicit solvent simulations the proper dielectric constant of the solute is a controversial issue in the literature (see, e.g., [46]).

However, MM-PB/GBSA is a technique mainly used for predicting relative binding energies and not absolute ones, since several effects such as hydration/dehydration, entropy and binding pathway contributions can hardly be taken into account. Thus the dielectric constant becomes only an important factor when the ranking of potential ligands is impacted. This is in particular the case when partial charges differ largely among the ligands to be ranked.

By definition, the concept of a dielectric constant is used to describe the collective behavior of matter and does not describe effects on the atomic level. In practice, using low dielectric constants for the solute (protein), such as $\epsilon=2$, accounts for electronic polarizability and is more sensitive to changes in the molecules, and can therefore be very useful for distinguishing between rather similar ligands. Nonetheless, as we point out with this study, the standard employment of such low dielectric constants may lead to wrong assumptions on the relative ranking of ligands, particularly when they differ in charges. Special care needs to be taken to adjust the dielectric constant for the system under investigation in order to avoid artefacts (e.g. contributions from polar residues located far from the binding site). Based on this study we suggest the use of a rather elevated solute dielectric constant of about 8 for kinases, in particular when investigating charged ligands.

\section{REFERENCES}

[1]T. P. Straatsma and H. J. C. Berendsen. Free energy of ionic hydration: Analysis of a thermodynamic integration technique to evaluate free energy differences by molecular dynamics simulations. The Journal of Chemical Physics, 89(9):58765886, November 1988

[2]Agastya P. Bhati, Shunzhou Wan, David W. Wright, and Peter V. Coveney. Rapid, Accurate, Precise, and Reliable Relative Free Energy Prediction Using Ensemble Based Thermodynamic Integration. Journal of Chemical Theory and Computation, 13(1):210-222, January 2017.

[3]Francesco Manzoni and Ulf Ryde. Assessing the stability of free-energy perturbation calculations by performing variations in the method. Journal of Computer-Aided Molecular Design, 32(4):529-536, 2018.

[4]Johan qvist, Carmen Medina, and Jan-Erik Samuelsson. A new method for predicting binding affinity in computer-aided drug design. Protein Engineering, Design and Selection, 7(3):385-391, March 1994.

[5]Peter A. Kollman, Irina Massova, Carolina Reyes, Bernd Kuhn, Shuanghong Huo, Lillian Chong, Matthew Lee, Taisung Lee, Yong Duan, Wei Wang, Oreola Donini, Piotr Cieplak, Jaysharee Srinivasan, David A. Case, and Thomas E. Cheatham. Calculating Structures and Free Energies of Complex Molecules: Combining Molecular Mechanics and Continuum Models. Accounts of Chemical Research, 33(12):889-897, December 2000.

[6]Samuel Genheden and Ulf Ryde. Comparison of end-point continuum-solvation methods for the calculation of protein-ligand binding free energies. Proteins, 80(5):1326-1342, May 2012.

[7]W. Wang, J. Wang, and P. A. Kollman. What determines the van der Waals coefficient beta in the LIE (linear interaction energy) method to estimate binding free energies using molecular dynamics simulations? Proteins, 34(3):395-402,
February 1999.

[8]Samuel Genheden and Ulf Ryde. The MM/PBSA and MM/GBSA methods to estimate ligand-binding affinities. Expert Opinion on Drug Discovery, 10(5):449461, May 2015.

[9]Aaron Weis, Kambiz Katebzadeh, Pr Sderhjelm, Ingemar Nilsson, and Ulf Ryde. Ligand affinities predicted with the MM/PBSA method: dependence on the simulation method and the force field. Journal of Medicinal Chemistry, 49(22):6596-6606, November 2006.

[10]Tingjun Hou, Junmei Wang, Youyong Li, and Wei Wang. Assessing the performance of the molecular mechanics/Poisson Boltzmann surface area and molecular mechanics/generalized Born surface area methods. II. The accuracy of ranking poses generated from docking. Journal of Computational Chemistry, 32(5):866-877, April 2011. 00234.

[11]Huiyong Sun, Youyong Li, Mingyun Shen, Sheng Tian, Lei Xu, Peichen Pan, Yan Guan, and Tingjun Hou. Assessing the performance of MM/PBSA and MM/GBSA methods. 5. Improved docking performance using high solute dielectric constant MM/GBSA and MM/PBSA rescoring. Physical chemistry chemical physics: PCCP, 16(40):22035-22045, October 2014. 00071.

[12]Tingjun Hou, Junmei Wang, Youyong Li, and Wei Wang. Assessing the performance of the MM/PBSA and MM/GBSA methods. 1. The accuracy of binding free energy calculations based on molecular dynamics simulations. Journal of Chemical Information and Modeling, 51(1):69-82, January 2011.

[13]Salla I. Virtanen, Sanna P. Niinivehmas, and Olli T. Pentikinen. Case-specific performance of MM-PBSA, MM-GBSA, and SIE in virtual screening. Journal of Molecular Graphics \& Modelling, 62:303-318, November 2015. 00005.

[14]Marc Adler and Paul Beroza. Improved ligand binding energies derived from molecular dynamics: replicate sampling enhances the search of conformational space. Journal of Chemical Information and Modeling, 53(8):2065-2072, August 2013. 00008.

[15]Krishna Ravindranathan, Julian Tirado-Rives, William L. Jorgensen, and Cristiano R. W. Guimares. Improving MM-GB/SA Scoring through the Application of the Variable Dielectric Model. Journal of Chemical Theory and Computation, 7(12):3859-3865, December 2011. 00015.

[16]Geoffrey T. Gibney and Jonathan S. Zager. Clinical development of dabrafenib in BRAF mutant melanoma and other malignancies. Expert Opinion on Drug Metabolism \& Toxicology, 9(7):893-899, July 2013.

[17]Anita D. Ballantyne and Karly P. Garnock-Jones. Dabrafenib: first global approval. Drugs, 73(12):1367-1376, August 2013.

[18]Alexander M. Menzies and Georgina V. Long. Dabrafenib and trametinib, alone and in combination for BRAF-mutant metastatic melanoma. Clinical Cancer Re search: An Official Journal of the American Association for Cancer Research, 20(8):2035-2043, April 2014.

[19]Lauretta Odogwu, Luckson Mathieu, Gideon Blumenthal, Erin Larkins, Kirsten B. Goldberg, Norma Griffin, Karen Bijwaard, Eunice Y. Lee, Reena Philip, Xiaoping Jiang, Lisa Rodriguez, Amy E. McKee, Patricia Keegan, and Richard Pazdur. FDA Approval Summary: Dabrafenib and Trametinib for the Treatment of Metastatic Non-Small Cell Lung Cancers Harboring BRAF V600e Mutations. The Oncologist, 23(6):740-745, June 2018.

[20]Weijiang Zhang. BRAF inhibitors: the current and the future. Current Opinion in Pharmacology, 23:68-73, August 2015.

[21]Cathrine L. Denton, Elisabeth Minthorn, Stanley W. Carson, Graeme C. Young, Lauren E. Richards-Peterson, Jeffrey Botbyl, Chao Han, Royce A. Morrison, Samuel C. Blackman, and Daniele Ouellet. Concomitant oral and intravenous pharmacokinetics of dabrafenib, a BRAF inhibitor, in patients with BRAF V600 mutation-positive solid tumors. Journal of Clinical Pharmacology, 53(9):955961, September 2013.

[22]Alicja Puszkiel, Galle No, Audrey Bellesoeur, Nora Kramkimel, Marie-Nolle Paludetto, Audrey Thomas-Schoemann, Michel Vidal, Franois Goldwasser, Etienne Chatelut, and Benoit Blanchet. Clinical Pharmacokinetics and Pharmacodynamics of Dabrafenib. Clinical Pharmacokinetics, 58(4):451-467, April 2019.

[23]A. Sali and T. L. Blundell. Comparative protein modelling by satisfaction of spatial restraints. Journal of Molecular Biology, 234(3):779-815, December 1993. 09586.

[24]Oliver Korb, Thomas Sttzle, and Thomas E. Exner. Empirical Scoring Functions for Advanced ProteinLigand Docking with PLANTS. Journal of Chemical Information and Modeling, 49(1):84-96, January 2009. 00321.

[25]Lin Li, Chuan Li, Subhra Sarkar, Jie Zhang, Shawn Witham, Zhe Zhang, Lin Wang, Nicholas Smith, Marharyta Petukh, and Emil Alexov. DelPhi: a comprehensive suite for DelPhi software and associated resources. BMC biophysics, 5:9, May 2012. 
[26]Lin Li, Chuan Li, Zhe Zhang, and Emil Alexov. On the Dielectric "Constant" of Proteins: Smooth Dielectric Function for Macromolecular Modeling and Its Implementation in DelPhi. Journal of Chemical Theory and Computation, 9(4):2126-2136, April 2013.

[27]Berk Hess, Carsten Kutzner, David van der Spoel, and Erik Lindahl. GROMACS 4: Algorithms for Highly Efficient, Load-Balanced, and Scalable Molecular Simulation. Journal of Chemical Theory and Computation, 4(3):435-447, March 2008. 07109.

[28]Junmei Wang, Wei Wang, Peter A. Kollman, and David A. Case. Automatic atom type and bond type perception in molecular mechanical calculations. Journal of Molecular Graphics \& Modelling, 25(2):247-260, October 2006. 01693.

[29]D. A. Case, D. S. Cerutti, T. E. Cheatham, T. A. Darden, R. E. Duke, T. J. Giese, H. Gohlke, A. W. Goetz, D. Greene, N. Homeyer, S. Izadi, A. Kovalenko, T. S. Lee, S. LeGrand, P. Li, C. Lin, J. Liu, T. Luchko, R. Luo, D. Mermelstein, K. M. Merz, G. Monard, H. Nguyen, I. Omelyan, A. Onufriev, F. Pan, R. Qi, D. R. Roe, A. Roitberg, C. Sagui, C. L. Simmerling, W. M. Botello-Smith, J. Swails, R. C. Walker, J. Wang, R. M. Wolf, X. Wu, L. Xiao, D. M. York, and P. A. Kollman. Amber 2017, University of California, San Francisco, 2017. 00000.

[30]Rashmi Kumari, Rajendra Kumar, Open Source Drug Discovery Consortium, and Andrew Lynn. g_mmpbsa-a GROMACS tool for high-throughput MM-PBSA calculations. Journal of Chemical Information and Modeling, 54(7):1951-1962, July 2014.

[31]Eric F. Pettersen, Thomas D. Goddard, Conrad C. Huang, Gregory S. Couch, Daniel M. Greenblatt, Elaine C. Meng, and Thomas E. Ferrin. UCSF Chimera-a visualization system for exploratory research and analysis. Journal of Computational Chemistry, 25(13):1605-1612, October 2004.

[32]Schrdinger, LLC. The PyMOL Molecular Graphics System, Version 1.8. November 2015.

[33]Gerd Neudert and Gerhard Klebe. DSX: a knowledge-based scoring function for the assessment of protein-ligand complexes. Journal of Chemical Information and Modeling, 51(10):2731-2745, October 2011.

[34]Melanie Schneider, Jean-Luc Pons, William Bourguet, and Gilles Labesse. Towards accurate high-throughput ligand affinity prediction by exploiting structural ensembles, docking metrics and ligand similarity. Bioinformatics (Oxford, England), July 2019.

[35]Jean-Luc Pons and Gilles Labesse. @TOME-2: a new pipeline for comparative modeling of proteinligand complexes. Nucleic Acids Research, 37(suppl_2):W485-W491, July 2009.

[36]David A. Bershas, Daniele Ouellet, Donna B. Mamaril-Fishman, Noelia Nebot, Stanley W. Carson, Samuel C. Blackman, Royce A. Morrison, Jerry L. Adams, Kristen E. Jurusik, Dana M. Knecht, Peter D. Gorycki, and Lauren E. RichardsPeterson. Metabolism and disposition of oral dabrafenib in cancer patients: proposed participation of aryl nitrogen in carbon-carbon bond cleavage via decarboxylation following enzymatic oxidation. Drug Metabolism and Disposition: The Biological Fate of Chemicals, 41(12):2215-2224, December 2013.

[37]Harma Ellens, Marta Johnson, Sarah K. Lawrence, Cory Watson, Liangfu Chen, and Lauren E. Richards-Peterson. Prediction of the Transporter-Mediated DrugDrug Interaction Potential of Dabrafenib and Its Major Circulating Metabolites. Drug Metabolism and Disposition: The Biological Fate of Chemicals, 45(6):646656,2017

[38]Bo Yang, Adel Hamza, Guangju Chen, Yan Wang, and Chang-Guo Zhan Computational determination of binding structures and free energies of phosphodiesterase-2 with benzo[1,4]diazepin-2-one derivatives. The Journal of Physical Chemistry. B, 114(48):16020-16028, December 2010. 00022.

[39]Cristiano R. W. Guimares and Mario Cardozo. MM-GB/SA rescoring of docking poses in structure-based lead optimization. Journal of Chemical Information and Modeling, 48(5):958-970, May 2008. 00153.

[40]J. Wang, P. Morin, W. Wang, and P. A. Kollman. Use of MM-PBSA in reproducing the binding free energies to HIV-1 RT of TIBO derivatives and predicting the binding mode to HIV-1 RT of efavirenz by docking and MM-PBSA. Journal of the American Chemical Society, 123(22):5221-5230, June 2001. 00603.

[41]S. C. Harvey and P. Hoekstra. Dielectric relaxation spectra of water adsorbed on lysozyme. The Journal of Physical Chemistry, 76(21):2987-2994, October 1972.

[42]M. K. Gilson and B. H. Honig. The dielectric constant of a folded protein. Biopolymers, 25(11):2097-2119, November 1986.

[43]Gregory King, Frederick S. Lee, and Arieh Warshel. Microscopic simulations of macroscopic dielectric constants of solvated proteins. The Journal of Chemical Physics, 95(6):4366-4377, September 1991.

[44]Federico Fogolari, Alessandro Brigo, and Henriette Molinari. Protocol for MM/PBSA Molecular Dynamics Simulations of Proteins. Biophysical Journal, 85(1):159-166, July 2003.
[45]Ben Zhuo Lu, Wei Zu Chen, Cun Xin Wang, and Xiao-jie Xu. Protein molecular dynamics with electrostatic force entirely determined by a single Poisson-Boltzmann calculation. Proteins, 48(3):497-504, August 2002.

[46]C. N. Schutz and A. Warshel. What are the dielectric "constants" of proteins and how to validate electrostatic models? Proteins, 44(4):400-417, September 2001. 


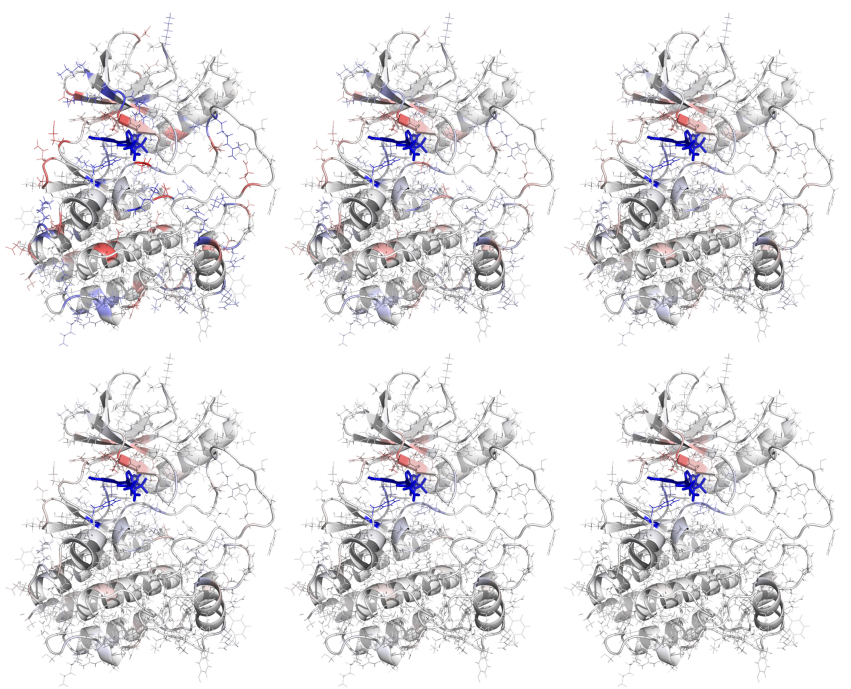

Figure S1. Energetic contribution of protein residues to ligand binding for the charged metabolite carboxy-dabrafenib (CDB) calculated with six different dielectric constants $\epsilon=(2,4,6)$ top row, and $\epsilon=(8,12,20)$ bottom row, from left to right. Color coding $=$ blue-white-red, with a minimum of -33 and a maximum of $+33 \mathrm{~kJ} / \mathrm{mol}$ (visualized with PyMol).

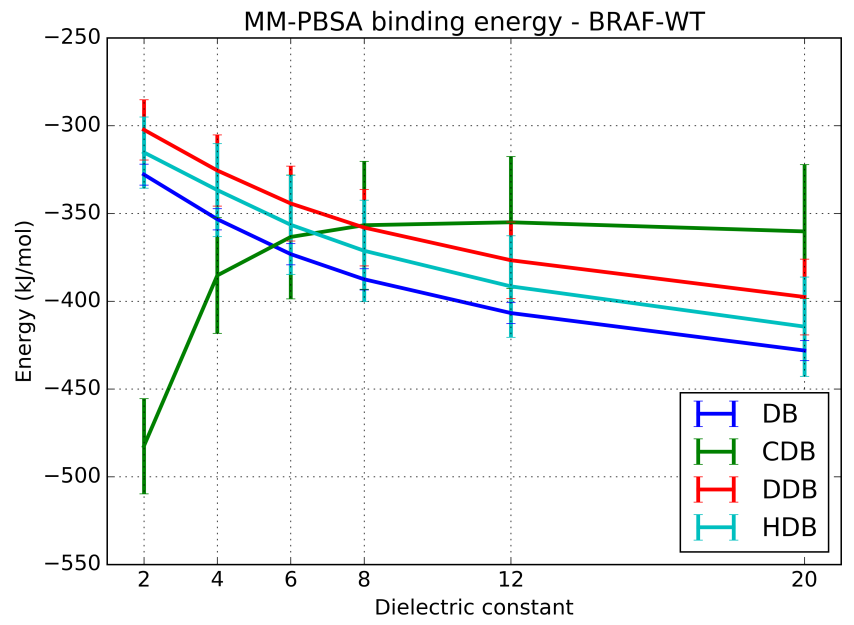

Figure S2. Averaged MM-PBSA binding energies (only enthalpic contribution) for BRAF-WT with dabrafenib (DB) and its metabolites carboxydabrafenib (CDB), desmethyl-dabrafenib (DDB), and hydroxy-dabrafenib (HDB). The averages are calculated for each ligand based on 2505 complex conformations from five replica MD trajectories at six different dielectric constants. The error bars are standard deviations across the five replica MD trajectories for each dielectric constant.

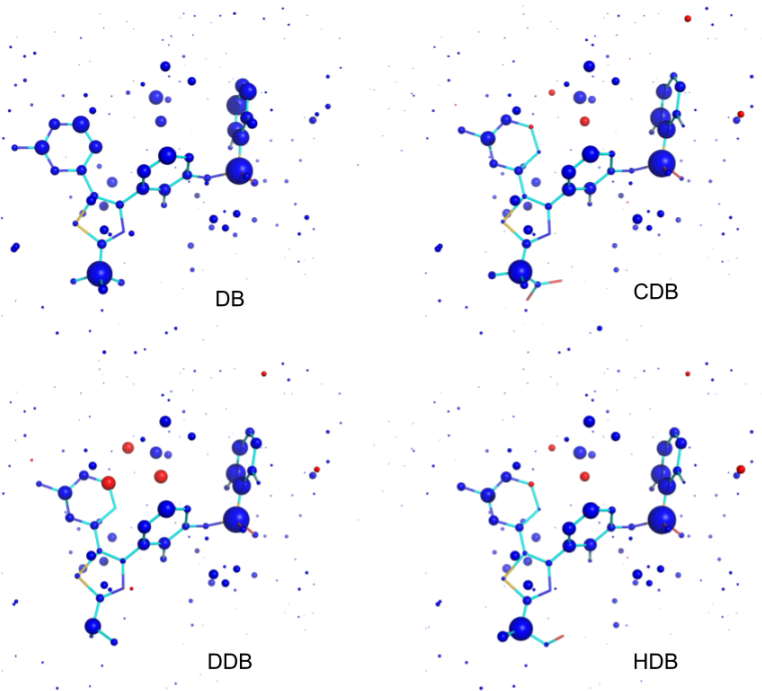

Figure S3. DSX evaluation: visualization of the per-atom score contributions of DB and its metabolites CDB, DDB and HDB in the binding pocket (visualized with PyMol). Favorably interacting atoms are surrounded by blue spheres and disfavorable interactions are shown in red. The sizes of the spheres correspond to the values of the contributing per-atom scores.

Listing 1. MM-PBSA example configuration file for g_mmpbsa with $\epsilon=6$ ; Polar calculation: "yes" or "no"

polar $=$ yes

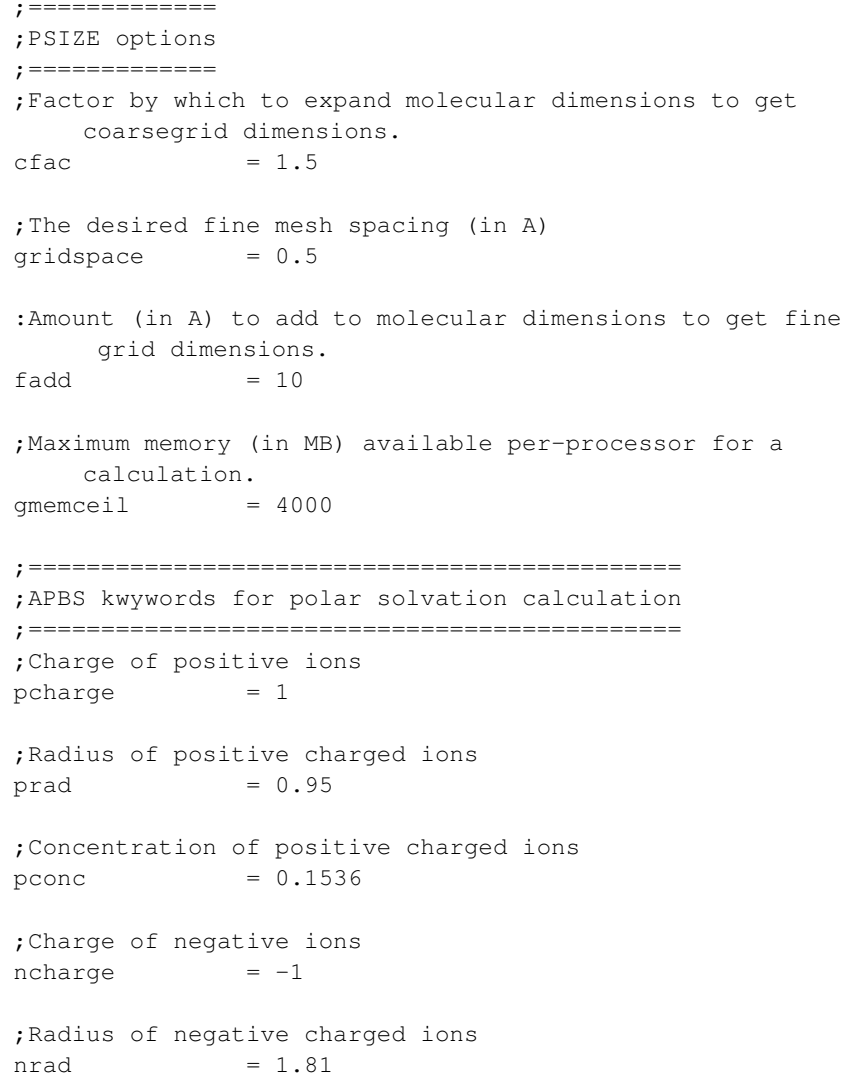


; Concentration of negative charged ions

nconc $\quad=0.1536$

; Solute dielectric constant

pdie $=6$

; Solvent dielectric constant

sdie $\quad=80$

; Reference or vacuum dielectric constant

vdie $\quad=1$

; Solvent probe radius

srad $\quad=1.4$

; Method used to map biomolecular charges on grid. chgm = splo or spl2 or spl4

chgm $\quad \operatorname{spl} 4$

; Model used to construct dielectric and ionic boundary. srfm $=$ smol or $\mathrm{spl} 2$ or $\mathrm{spl} 4$

srfm

$=\mathrm{smol}$

; Value for cubic spline window. Only used in case of srfm $=\operatorname{spl} 2$ or $\operatorname{spl} 4$

swin $\quad=0.30$

; Numebr of grid point per $A^{\wedge} 2$. Not used when $(\operatorname{srad}=0.0)$ or $(\operatorname{srfm}=\operatorname{spl} 2$ or $\operatorname{spl} 4)$

sdens $=10$

; Temperature in $\mathrm{K}$

temp $\quad=300$

; Type of boundary condition to solve $\mathrm{PB}$ equation. bcfl= zero or sdh or mdh or focus or map $\mathrm{bcfl}$

$$
=\mathrm{mdh}
$$

; Non-linear (npbe) or linear (lpbe) PB equation to solve PBsolver $=1 \mathrm{pbe}$ ; APBS kwywords for Apolar/Non-polar solvation calculation $;==========================================$
; Non-polar solvation calculation: "yes" or "no" apolar

$=$ yes

; Repulsive contribution to Non-polar

$;===$ SASA model $====$

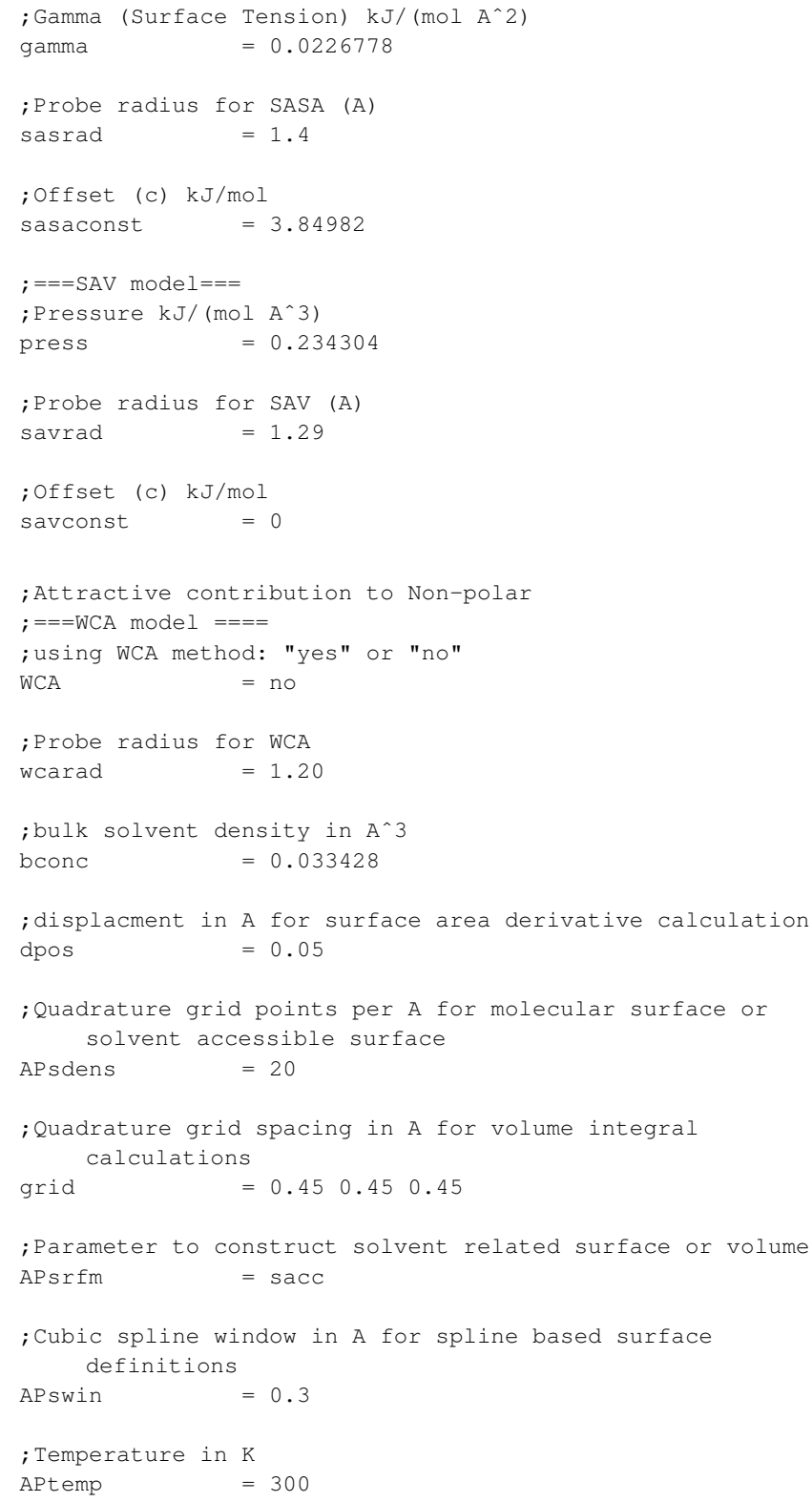

